\title{
Recurrence Quantity Analysis of the Instantaneous Pressure Fluctuation Signals in the Novel Tank with Multi-Horizontal Submerged Jets
}

\author{
H.-B. Meng, M.-Y. Song, Y.-F. Yu, ${ }^{*}$ and J.-H. Wu \\ School of Energy and Power Engineering, \\ Shenyang University of Chemical Technology
}

doi: 10.15255/CABEQ.2014.2043

Original scientific paper

Received: May 7, 2014

Accepted: February 19, 2016

The circulating jet tank (CJT) has been an alternative piece of equipment for mixing instead of the bottom-stirring tank, which is widely used in industrial applications. The recurrence plots (RPs) and recurrence quantification analysis (RQA) of pressure fluctuation signals (PFS) in the novel CJT were employed to reflect the chaotic extent of jet mixing. The recurrence rate, determinism and averaged diagonal line length of PFS were evaluated at different Reynolds numbers, radial positions and axial positions. The profiles of recurrence rate, determinism and averaged diagonal line length had similar tendency with the increasing Re, which showed that the determinism of PFS increased and the randomness of the chaotic system became small. With the increase in $z / H$, the recurrence characteristics of PFS at $\theta_{\mathrm{m}}=\pi / 6$ gradually increased, which were smaller than that of other $\theta_{\mathrm{m}}$. The results of this study provide a deep understanding of the hydrodynamics in the CJT, and thus lay a foundation for further design optimization.

Key words:

average diagonal line length, CJT, determinism, PFS, RPs, recurrence rate

\section{Introduction}

Jet mixers have numerous advantages over impellers, which makes them suitable for industrial applications, such as extraction ${ }^{1}$, chemical reaction ${ }^{2}$, absorption and desorption ${ }^{3}$ mixing $^{4}$, reaction injection molding ${ }^{5}$, side-dump combustion ${ }^{6-7}$, etc. There have been many studies on jet mixing in the past 70 years. Commonly encountered jet mixing tanks use a single jet with constant mean flow. Fossett and Prosser $^{8}$ determined the performance of free jets for mixing fluids in large circular tanks with scale models. Lehrer ${ }^{9}$ defined the entrainment ratio in the fully developed jet, and found that the jet mixing time was inversely proportional to the entrainment ratio. Unsteady jets have been found to be more energy-efficient than steady jets ${ }^{10}$. Riffat et al. ${ }^{11}$ investigated the refrigerant flow patterns and pressure distribution through the ejector unit using the CFD method. Ranade ${ }^{12}$ investigated the flow patterns and mixing performances in jet mixing tanks using the standard $\kappa-\varepsilon$ model. Simulations of mixing performance in various vessel configurations have been conducted as shown in the References of Jayanti ${ }^{13}$, Zughbi and Rakib ${ }^{14}$. Patwardhan and Thatte ${ }^{15}$ investigated the effects of jet velocity, nozzle clearance, liquid depth, and tank size on mixing time with the help of CFD modeling. Some efforts on computational fluid dynamics and experimental studies of mixing in fluid-jet-agitated tanks have been made ${ }^{16-21}$.

${ }^{*}$ Corresponding author: e-mail: taroyy@163.com
The circulating jet tank (CJT) is novel jet mixing equipment based on multi-horizontal submerged nozzles. The CJT has been used successfully in industrialized production processes of polyvinyl chloride (PVC) in Liaoning Huajin Chemical Industry Group Co., Ltd. for over 10 years, and has provided better economic benefits ${ }^{22}$. The steady flow in the CJT has been investigated based on numerical simulations by Yu et al. ${ }^{23}$ It was proved that the internal fluid flow of the jet tank was a strong shear turbulent jet. However, due to the complexity of the hydrodynamics, the design and scaling of this chemical reactor are still not straightforward with an inadequate understanding of fluid properties. The effects of global circulation, trailing vortices, and small-scale turbulence on macromixing and micromixing is not so clear. Some different types of motion coexist in the CJT: mean flow or global circulation, periodic fluctuations or vortices induced by the jet impinging the wall and the baffles, and the turbulent fluctuations that finally dissipate the kinetic energy ${ }^{24}$.

There are many other techniques that could be used to determine the hydrodynamic properties of CJT, such as Phase Doppler Particle Anemometer (PDPA), Particle Image Velocimetry (PIV), and Pressure Fluctuation Signals (PFS). In recent years, a number of methods have been devised to analyze the dynamical characteristics of the time series ${ }^{25-29}$. The jet flow in the CJT was proved to be a nonlinear system based on PFS experimental investigation $^{22,30,31}$. The nonlinear systems are able to gener- 
ate complex signals, which cannot be effectively distinguished from noise using linear tools such as spectral or statistical analysis. Nonlinear time series analysis is a powerful theory, which could be used to extract the characteristic quantities of a particular system solely by analyzing the time course of one of its variables. Thus, the theory of nonlinear time series analysis offers tools that bridge the gap between the experimentally observed irregular behavior and the deterministic chaos theory ${ }^{32}$. Recurrence is a fundamental characteristic of many dynamical systems introduced by Poincaré in 1890. In 1987, Eckmann et al..$^{33}$ introduced the method of recurrence plots (RPs) to visualize the recurrences of dynamical systems. Applications of RPs can be found in numerous fields of research, such as astrophysics, earth sciences, engineering, biology, cardiology or neuroscience ${ }^{34,35}$. The different aspects of recurrences can be inferred by measures of complexity which quantify the small-scale structures in RPs, known as recurrence quantification analysis (RQA). These measures are based on the recurrence point density, and the diagonal and vertical line structures $^{29}$. In this paper, a combination of RPs and RQA was used to identify and characterize the fluid dynamics of PFS in the novel CJT.

\section{Methodology}

For deterministic dynamical systems, including the nonlinear and chaotic systems, all can be defined as a recurrence state where one state is very similar to the other state ${ }^{36}$. The recurrence state can be drawn in a two-dimensional plane through a time series reconstructed in the phase space:

$R_{\mathrm{i}, \mathrm{j}}^{\mathrm{m}}=\Theta\left(\varepsilon-\left\|\vec{x}_{i}-\vec{y}_{j}\right\|\right), \quad \vec{x}_{i} \in R^{\mathrm{m}}, \quad i, j=1, \cdots, N$

where $N$ is the number of the considered states of $x_{\text {; }}$, $\varepsilon=\alpha \cdot$ std is the threshold of distance, $\alpha$ is the radius to be selected, std is the standard deviation of the time series. $\|\bullet\|$ is a maximum or Euclidean norm, and $\Theta(\bullet)$ is the Heaviside function.

Its overall structural characeristics and structure of detailed texture can be used to describe the characteristics of different system states. RPs offer the possibility to easily find and assess extreme and rare events by using the frequency of their recurrences. The graphical representation of RPs may be complicated to evaluate, since they are considered as qualitative tools to detect hidden rhythms graphically. The method of RQA developed by Zbilut and Webber ${ }^{37-38}$ has been widely used in the analysis of chaotic time series, which describes the structure character in recurrence plots. The important advantage of the method based on the quantification of RPs is that the required data length can be relatively short.
DET represents the percentage of recurrent points belonging to a diagonal line of a minimum length of $l_{\min }=2$, and $p(l)$ denotes the probability of finding a diagonal line of length $l$ in the $R P$.

$$
D E T=\left(\sum_{l=l_{\min }}^{N-1} l \cdot p(l)\right) / \sum_{i, j=1}^{N} R_{\mathrm{i}, \mathrm{j}}
$$

$R R$ is the percentage of points that are recurrent, which denotes the rate between the number of points close to each other and all pairs of points in m-dimensional phase space.

$$
R R=\frac{1}{N^{2}} \sum_{i, j=1}^{N} R_{\mathrm{i}, \mathrm{j}}
$$

Because the $R R$ reflects the density of recurrence points in the RP, the more the dynamic system is random, the lower is the $R R^{39}$.

The ratio between $D E T$ and $R R$ is:

$$
R A T I O=D E T / R R
$$

The maximum diagonal line length, $L_{\max }$, is calculated from the formula:

$$
L_{\max }=\max \left(\left\{l_{i} ; i=1 \cdots N_{l}\right\}\right)
$$

Divergence $D I V$ is the inverse of $L_{\max }$, which is related with the $\mathrm{K}$ entropy of the system, i.e. with the sum of the positive Lyapunov exponents.

$$
D I V=1 / L_{\max }
$$

The Shannon entropy (ENTR) of the diagonal line lengths $p(l)$ provides a measure of the complexity of RPs. ENTR is computed from the formula:

$$
\begin{gathered}
E N T R=-\sum_{l=l_{\min }}^{N} P(l) \ln P(l) \\
P(l)=p(l) / \sum_{l=l_{\min }}^{N} p(l)
\end{gathered}
$$

where $P(l)$ is the individual probability of a diagonal line occurring with length $l$. The higher the entropy value, the clearer and the more complete the system structure.

An average diagonal line length of $L$ is defined as

$$
L=\left(\sum_{l=l_{\min }}^{N-1} l \cdot p(l)\right) / \sum_{l=l_{\min }}^{N-1} p(l)
$$

which characterizes the average time that two segments of the trajectory are close to each other, and can be interpreted as the mean prediction time ${ }^{40}$. 


\section{Experiments}

The experimental apparatus mainly consisted of the circulating jet system and high-speed data acquisition system, as shown in Fig. 1. The number of horizontal jet nozzles distributed with a uniform space in the four vertical risers was $\mathrm{N}_{j}=32$. The online dynamic data acquisitions were implemented based on dynamic signal collector DEWE-3021 and five dynamic pressure sensors DYG32000. A WILO MHI802 centrifugal pump was used to withdraw fluid from the down-comer and pump it through the inlet pipe to the jet nozzles with a diameter of $d_{\mathrm{j}}=$ $0.003 \mathrm{~m}$, where the fluid was ejected into the tank as the high-speed jet stream.

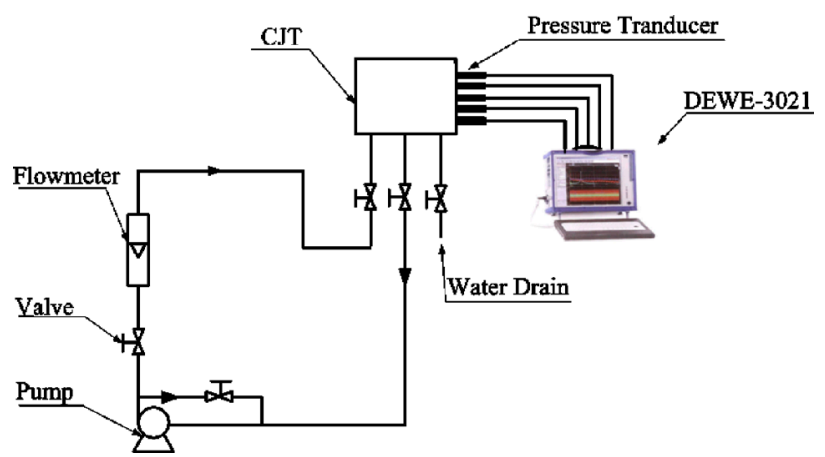

Fig. 1 - Schematic diagram of the experimental set-up for instantaneous PFS in the CJT

Distilled water was used as the experimental medium in order to ensure the precision of the experimental data and the sensitivity of the probes. The flow rate of the pump outlet was set as $Q=$ $2.78 \cdot 10^{-4}-2.5 \cdot 10^{-3} \mathrm{~m}^{3} \mathrm{~s}^{-1}$ to guarantee the fully turbulent fluid in all cases. The Reynolds number can be defined as equation

$$
\operatorname{Re}=\frac{4 \rho Q}{\pi d_{j} \mu N_{j}}
$$

where $d_{\text {. }}$ represents the diameter of the jet nozzles and $\mathrm{N}_{\mathrm{i}}$ denotes the number of jet nozzles. In addition, the density $\rho$ and kinematic viscosity $\mu$ of water are given as $\rho=998 \mathrm{~kg} \mathrm{~m}^{-3}$ and $\mu=1.003 \cdot 10^{-3}$ $\mathrm{Pa} \cdot \mathrm{s}$, respectively. The corresponding Re ranged from 3660 to 32940 .

The original sampling frequency of the PFS was $500 \mathrm{~Hz}$, satisfying the Nyquist criterion. The data length of the time series in each sample was 126000. The PFS measurement was repeated three times under each condition in order to ensure the repeatability of the experimental data. The experiment was conducted in a quarter of the CJT. Taking into the account the parameters of tank diameter and pipe thread adaptor and clamp nut of DYG32000 pressure sensor as described in Reference ${ }^{41}$, four typical circumferential positions were chosen. The group numbers of radial positions, axial positions, and circumferential positions were $10 \times 5 \times 4$, detailed information of the measurement points referred to the literature of Meng et al. ${ }^{22,41}$ The measurements of the PFS under different radial positions were accomplished by the scale on the probe.

\section{Results and discussion}

\section{Signal processing}

All obtained experimental data are more or less contaminated by noise based on the fact that noise and distortion are the main factors limiting the capacity of data and the accuracy of the results. Wavelet analysis as a better method has a particular advantage in eliminating noise of the signal and has wide application foreground. The Daubechies wavelets are compactly supported wavelets with external phase and highest number of vanishing moments for a given support width, which have been used in many systems ${ }^{42-45}$. Firstly, the PFS of CJT could be decomposed to obtain a time history of the different frequency bands using wavelet transform with Daubechies wavelets. Then, the wavelet transform modulus maxima suitable for non-stationary signal was selected to denoise the different frequency band PFS which was used to reconstruct the PFS with noise reduction.

The decomposition errors $\mathrm{E}$ between original and reconstructed PFS time series with Dau2-Dau10 at different scales are shown in Fig. 2. It could be observed from Fig. 2 that the wavelet decomposition errors of PFS with Dau2 were the least under different decomposition scales, while the decomposition errors with Dau9 were the largest, and those with Dau3 were the second largest. The values of $E$ increased with the increasing decomposition scale at the same Daubechies wavelet. It could be concluded that the decomposition errors have the similar variation tendency at different scales. That is to say, the decomposition errors firstly increase then decrease with an increase in $\theta$. The decomposition error of the PFS for $\theta_{\mathrm{m}}=5 \pi / 12$ is minimum with the same Daubechies wavelet and scale, while the maximum error exists for $\theta_{\mathrm{m}}=\pi / 4$. Thus, it is noted that attention should be paid to the effect of different factors related to Daubechies wavelet in order to extract the characteristic information of wavelet decomposition. As a result, Dau2 wavelet with 10 levels was chosen to denoise the instantaneous PFS.

All nonlinear methods of time series analysis are based on the state space reconstruction of the underlying system. Fig. 3 presented the 2D projections of the reconstructed phase space of original PFS time series. The transient fluctuations of original and reconstructed PFS time series are shown in Fig. 3(a) 

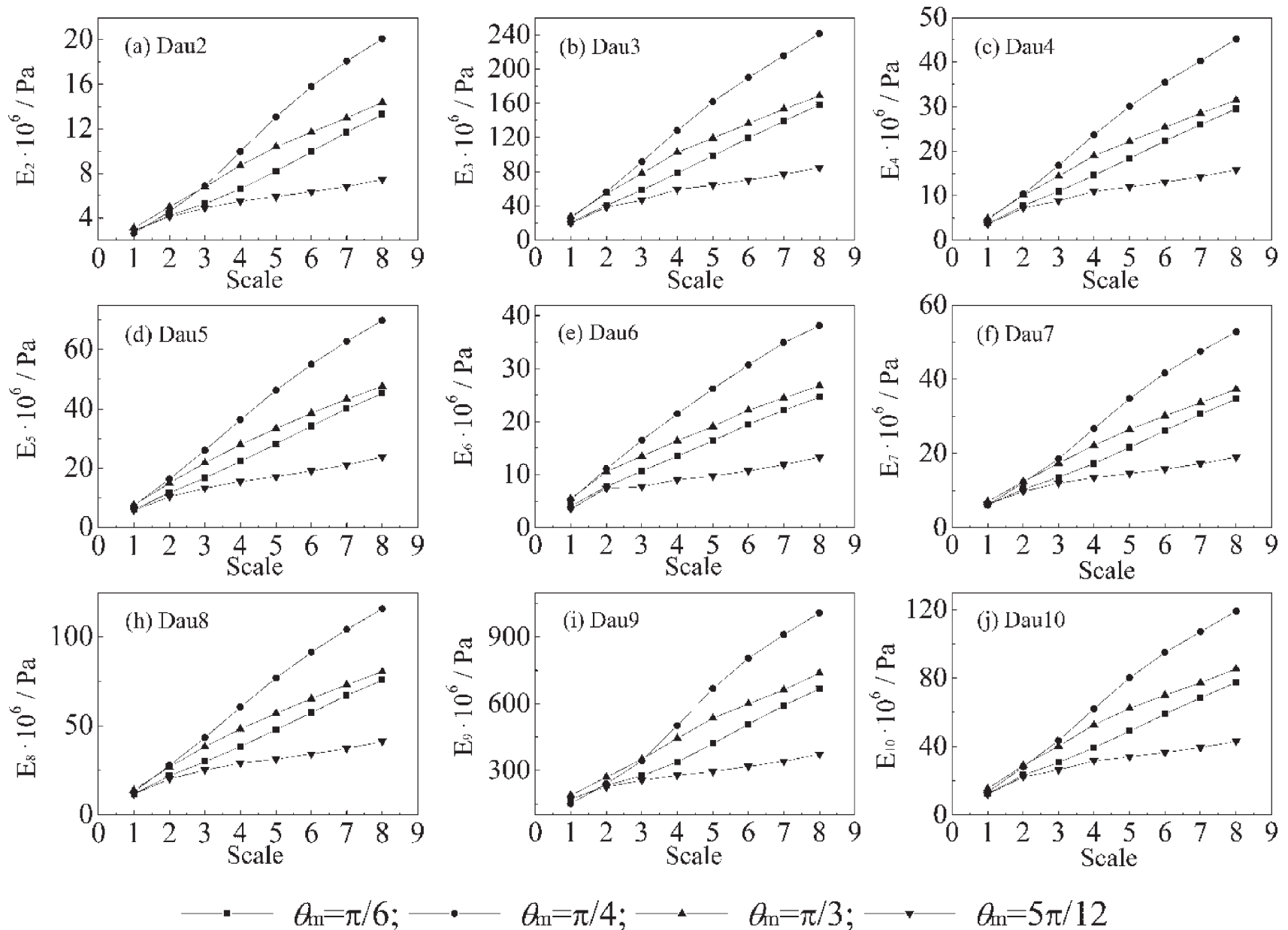

Fig. 2 - Decomposition errors between original and reconstructed PFS time series with different Daubechies wavelets and scales

and 3(c), respectively. Some outliers were effectively removed and the main developed tendency of PFS was reserved. It could be clearly observed that the two-dimensional attractors of phase space structure in Fig. 3(d) are much smoother and more obvious than those in Fig. 3(b), which additionally confirms the success of the noise reduction algorithm. The effect of the time delay on the attractor could not be identified for the existence of the noise, while the denoised signals based on wavelet were clearly visible, and the outline of the phase space was not altered during the noise reduction.

\section{Determining parameters for non-stationary series}

Over the last years, RPs and RQA have become quite popular in various branches of science ${ }^{46}$. The key issue in application of RPs and RQA is the selection of suitable parameters of the PFS under investigation. The choice of the neighborhood size is also still under discussion and often causes uncertainties in applying RPs and RQA. Several rules of thumb for the choice of the threshold have been suggested with a few percent of the maximum phase space diameter, a value which should not exceed $10 \%$ of the mean or the maximum phase space diameter, or a value that ensures a recurrence point density of approximately $1 \% 0^{47,48}$. Because the noise of the signal has a strong effect on the RP, the threshold value is sometimes set equal to 5 times the measurable noise standard deviation, widely adopted in many applications. If $\varepsilon$ is too small, recurrences mainly appear due to the fluctuations caused by the noise. If $\varepsilon$ becomes too large, almost every point is in the neighborhood of every other point, which may hide the characteristic recurrence structure.

In order to select a suitable radius, the influence of the radius on divergence was investigated based on a group of measured signals, as shown in Fig. 4. The phase space structure was reconstructed with the embedding parameters $\mathrm{m}=3$ and $\tau=3 \Delta t$ (fixed amount of nearest neighbours). The embedding parameters were estimated by the false nearest neighbours method for dimension, and the mutual information method for time delay ${ }^{27}$. It could be seen that the $D I V$, the inverse of $L_{\max }$, declines with increasing $\alpha$ value, which means that the determinacy of PFS in CJT is becoming stronger. The variation rate is below $11.5 \%$ for $\alpha \geq 0.6$. In order to keep sufficient continuous structures in the RP, $\alpha=0.7$ was selected to 

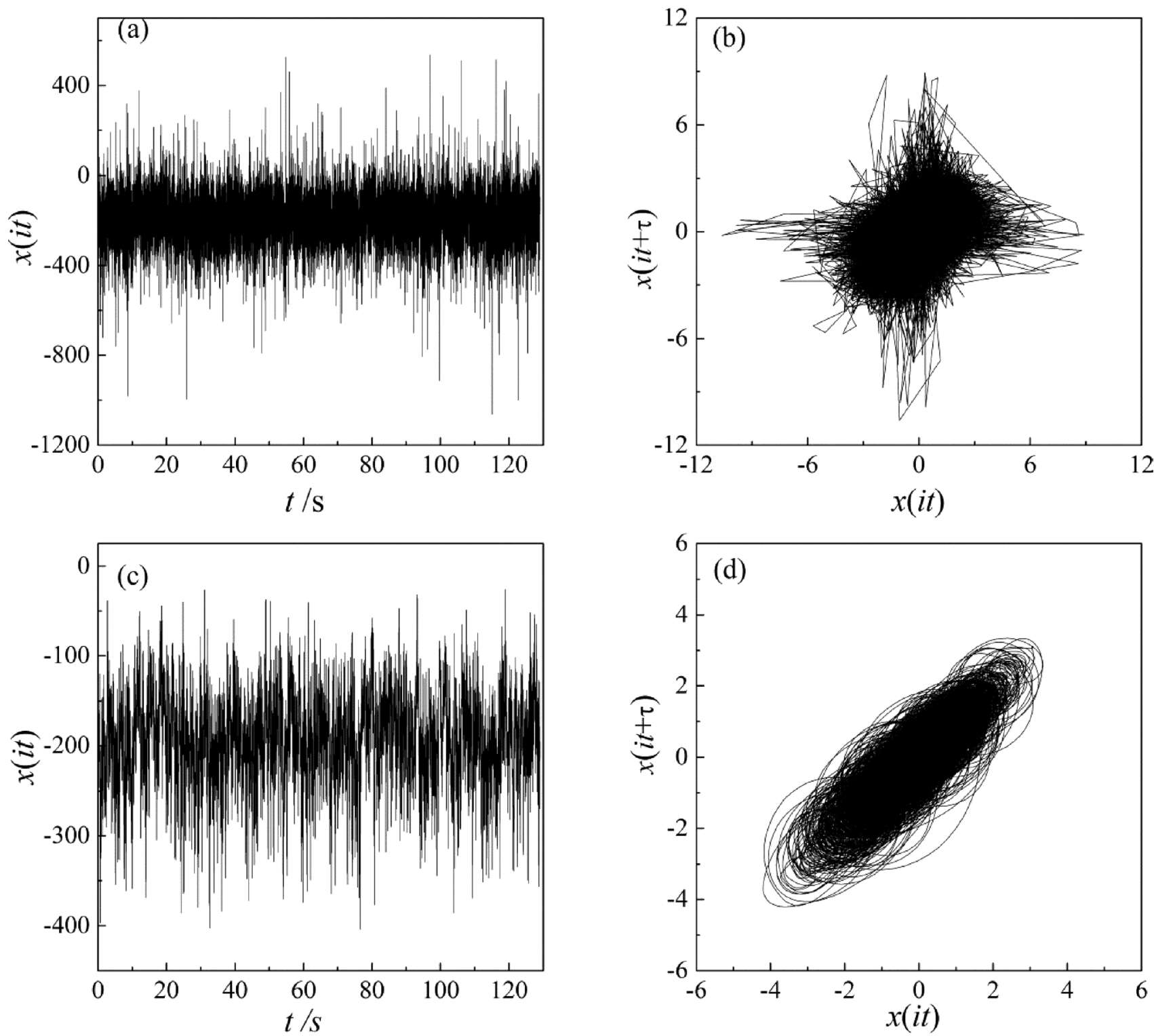

Fig. 3 - Comparison between original and reconstructed PFS time series. (a) original and (c) de-noising transient PFS, two-dimensional attractors of (b) original and (d) de-noised normalized PFS

analyse the recurrence characteristics of PFS in CJT in this study.

The instantaneous dynamics characteristics of PFS in CJT can be inspected through the RQA calculated in the consecutive epochs. The Recurrence Rate and Entropy of PFS in consecutive epochs are plotted as shown in Fig. 5. Compared with the RR values of the stochastic and periodic systems as shown in Fig. 5(a), the chaotic dynamic behavior of CJT is between long-term predictable and completely unpredictable systems. In addition, RR values increase with an increase in $R e$. At this condition, the effect of macromixing on the PFS in the tank is dominant against meso- and micro-phenomena, and the PFS approaches periodic behavior rather than completely unpredictable systems. It is ex-

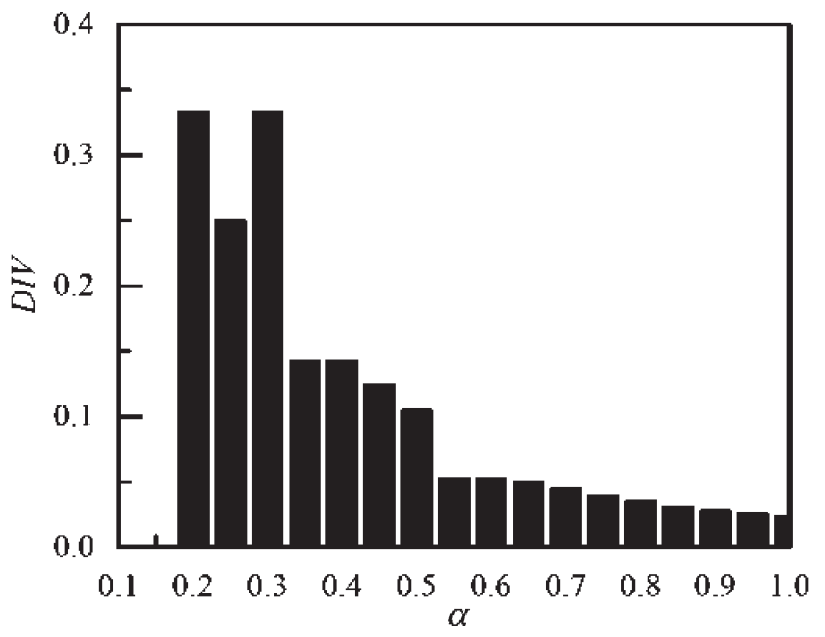

Fig. 4 - Divergence distribution of PFS in CJT with different threshold coefficients 

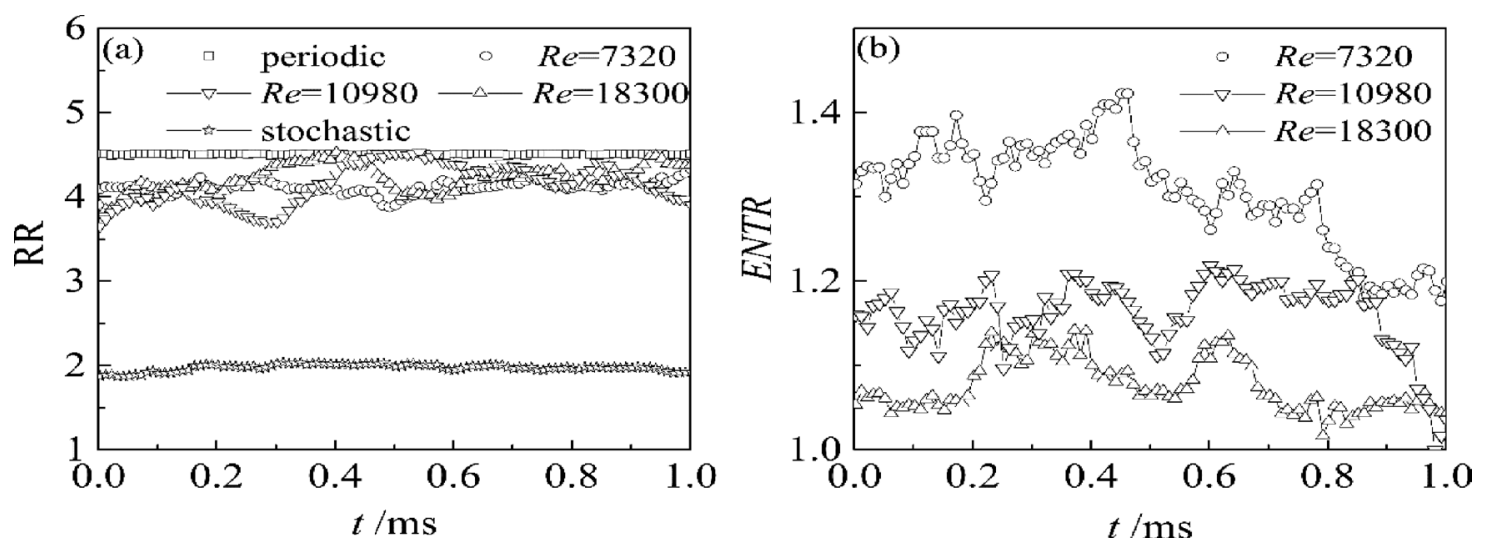

Fig. 5 - Recurrence rate and entropy of PFS at $r / R=0.825$ and different Re, epoch length $=1000 ; N=2000 ; \alpha=0.7 ; l_{\min }=2$

pected that a more periodic system has lower entropy, which can be confirmed by the plot of entropy values. As illustrated in Fig. 5(b), the entropy is smaller in higher Re, which reveals that the contribution of larger scale secondary flow structure beomes more important in higher $R e$ and reduces the complexity of turbulent flow.

\section{The RPs of the instantaneous PFS under different Reynolds numbers}

The recurrence plots of PFS at $r / R=0.895$ of 113 under different Reynolds numbers are shown in Fig. 6. It can be seen that the Reynolds number has impact on the structure of detailed texture. With an increase in $R e$, the structures of RPs transfer from the dispersed and isolated random point to the rectangular massive segments with developed line structure along the main diagonal. That is to say, the character of recurrence gets better and the predictability becomes high with an increase in $R e$. For $R e<18300$ the RP points distribute homogeneously, whereas the diagonal line segments parallel to the main diagonal are present for $R e \geq$ 18300.

The effect of Reynolds numbers on RQA variables, such as DET, RR, $L$ and RATIO of PFS at three radial positions of $\mathrm{d} 13$ in the CJT are presented in Fig. 7. It can be concluded that four characteristic paremeters have the similar variation trends with the increasing $R e$, which indicates that the recurrence character gradually increases with better periodicity and good stationeriness. It is found from Fig. 7 that the values of $D E T$ and $R R$ slowly increase, but the values of $L$ decrease with the increase in $R e=7320-10980$. This means that the recurrence characteristic is little affected by the Reynolds number under low $R e$. The Reynolds number has an influence on the RQA variables of $r / R=0.685$ for $R e \geq 18300$. The RQA parameters significantly increase and then smoothly change with the increasing $R e$. The reason being that a pair of strong vorticities are formed after the boundary fluid of jet impinging the tank wall and the baffle, which strengthens the macro-scale flow structure of the zone ${ }^{24}$.

\section{The RPs of the instantaneous PFS under different radial positions}

The distributions of $D E T, R R$ and $L$ at different $r / R$ of $\mathrm{d} 8$ for $R e=32940$ are plotted in Figs. 8-10. It can be seen that the three variables aforementioned have the same variation trend with an increase in $r / R$ for given $\theta_{\mathrm{m}}$ and $R e$. The variables at $\theta_{\mathrm{m}}=\pi / 6$ firstly decrease and then increase with the increasing $r / R$ which have the maximum values compared with those at other $\theta_{\mathrm{m}}$ for $R e=7320$. There are many differences between RQA variables of different radial positions for $R e=32940$. The variables have the minima with $\theta_{\mathrm{m}}=\pi / 6$, which suggests that the PFS at $\theta_{\mathrm{m}}=\pi / 6$ have higher stability for the initial developed region of jet. The randomness of PFS at $\theta_{\mathrm{m}}=\pi / 6$ for $R e=32940$ is larger than that for $R e=7320$. The recursion characteristics decrease firstly, then sharply increase and decrease at last with the increasing $r / R$ at other $\theta_{\mathrm{m}}$ in high turbulent flow. The radial positions of the maximum are different among the different $\theta_{\mathrm{m}}$. That is, the strong determinacy are in $r / R=0.755$ for $\theta_{\mathrm{m}}=\pi / 4$, $r / R=0.895$ for $\theta_{\mathrm{m}}=\pi / 3$, and $r / R=0.685$ for $\theta_{\mathrm{m}}=5 \pi / 12$, respectively.

It could be also concluded from Figs. 8-10 that there are higher recurrence characteristics at $r / R=$ 0.685 and $r / R=1.0$ for $R e=7320$ because of the coupling effect between jet flow and secondary wall jet induced by the jet impinging the baffle and tank walls. The maximum of $D E T$ and $R R$ for $r / R=0.685-0.755$ is at $\theta_{\mathrm{m}}=\pi / 4$, while it is for $r / R=0.755-1$ at $\theta_{\mathrm{m}}=\pi / 3$. The maximum of $L$ for $r / R=0.685-0.79^{\mathrm{m}}$ is at $\theta_{\mathrm{m}}=\pi / 4$, while it is for $r / R=0.79-1$ at $\theta_{\mathrm{m}}=\pi / 3$. And the values at $\theta_{\mathrm{m}}=$ $5 \pi / 12$ are always in the middle between $\theta_{\mathrm{m}}=\pi / 4$ and $\theta_{\mathrm{m}}=\pi / 3$ with different $r / R$. 


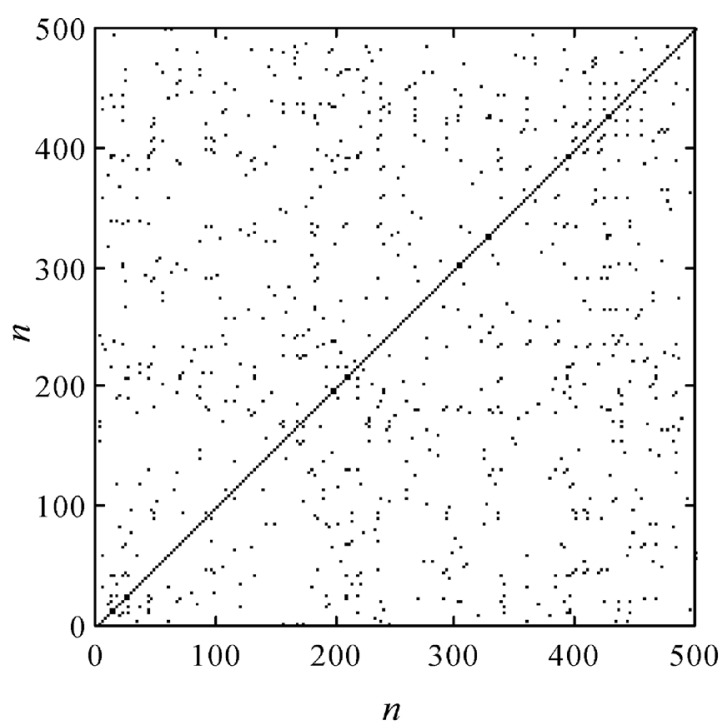

(a) $\operatorname{Re}=7320$

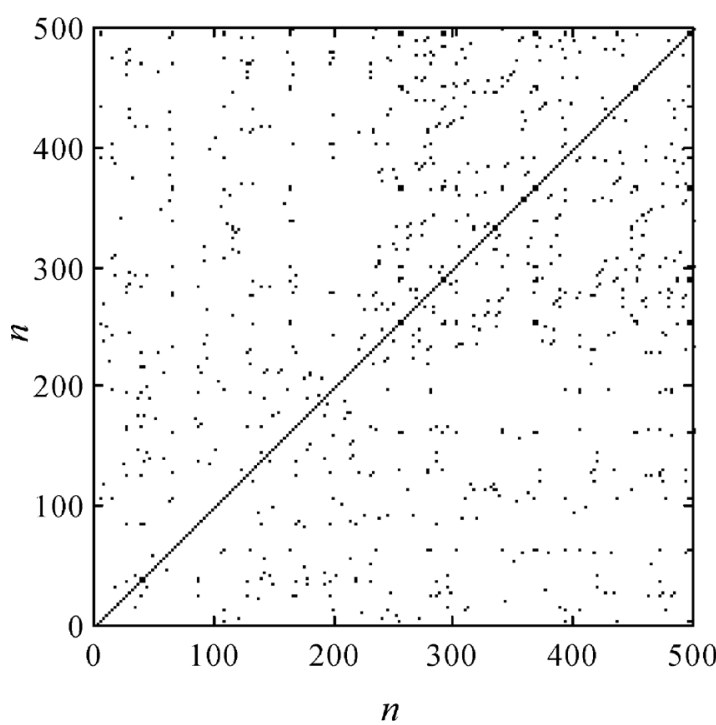

(c) $\operatorname{Re}=18300$

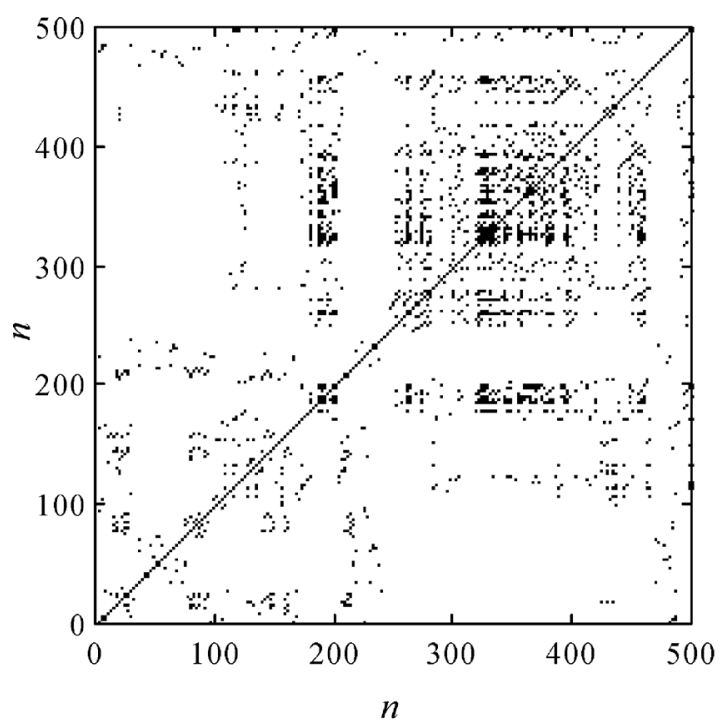

(e) $R e=29280$

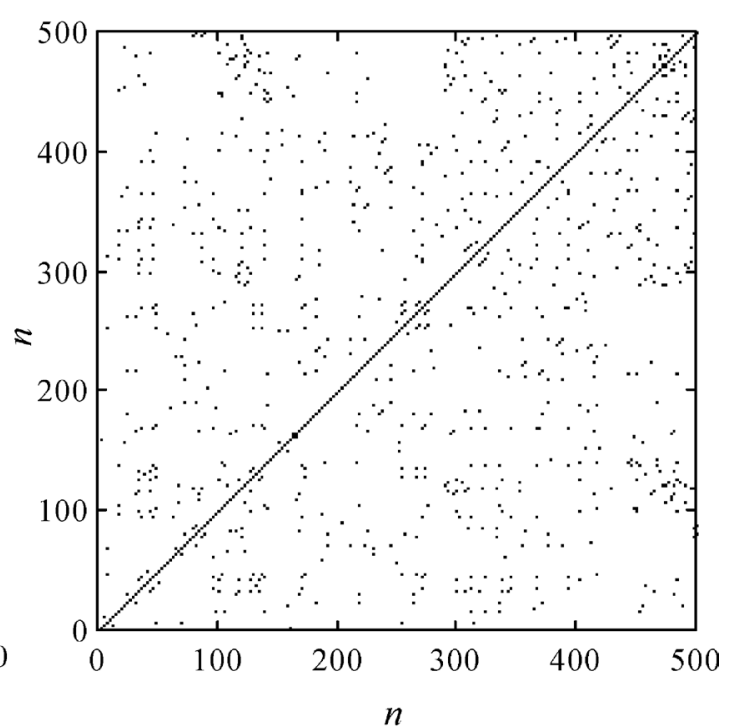

(b) $R e=10980$

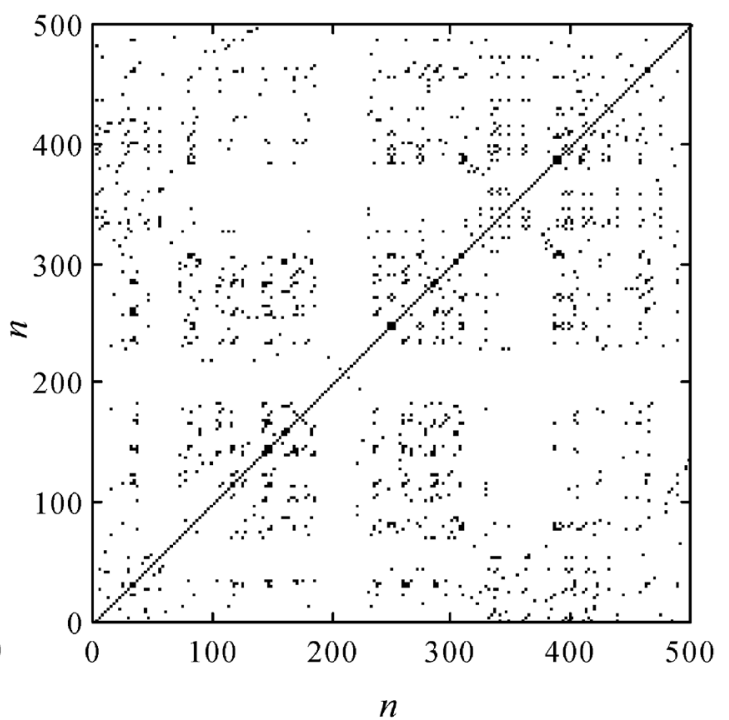

(d) $R e=25620$

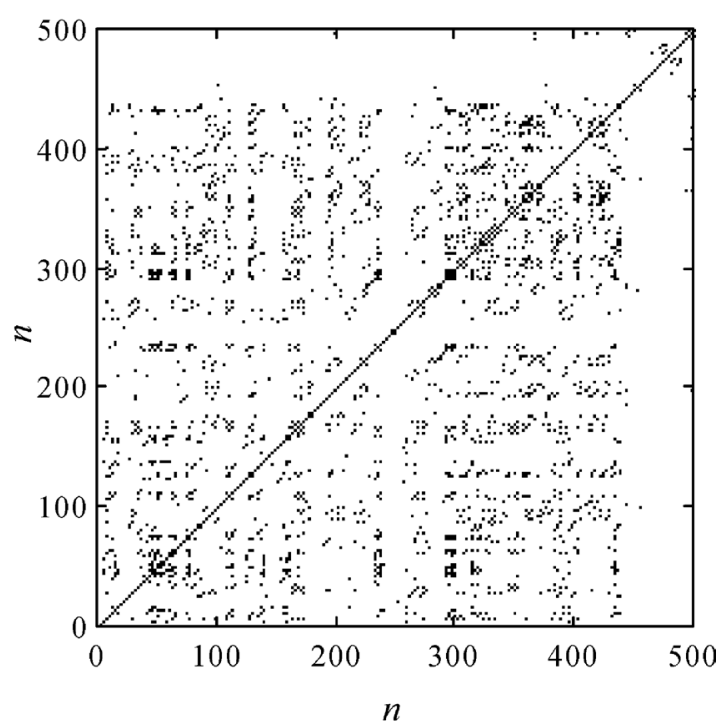

(f) $R e=32940$

Fig. 6 -Recurrence plot of PFS at different Re 

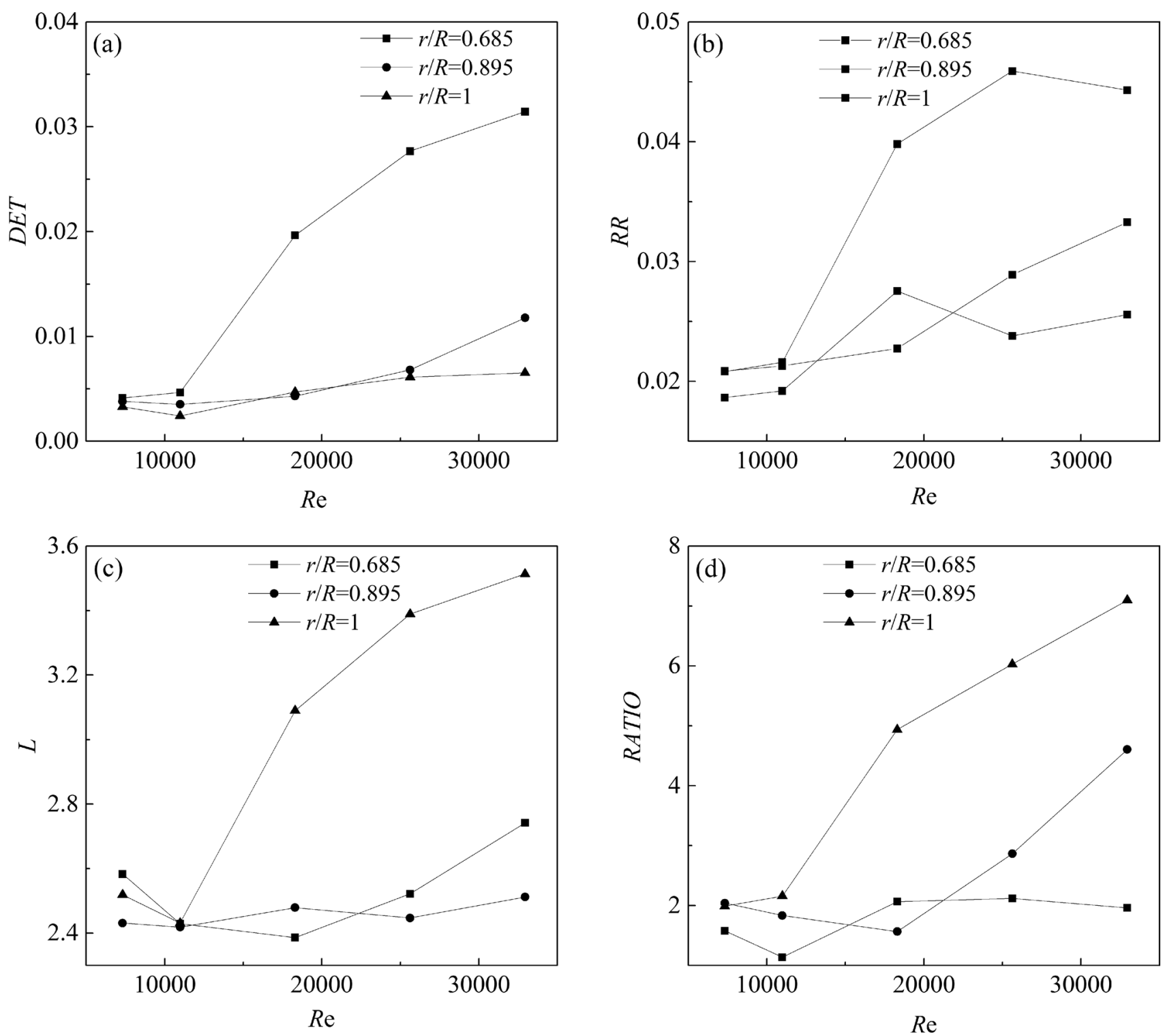

Fig. 7 -RQA variables of the PFS for different Re

\section{The RPs of the instantaneous PFS under different axial positions}

The three characteristic variables, i.e. DET, RR and L, of RPs at $r / R=0.895$ with an increase in axial positions $z / H$ are presented in Figs. 11-13. It could be seen that the recurrence characteristics of PFS at $\theta_{\mathrm{m}}>\pi / 6$ and $R e=7320$ vary little with an increase in $z / H$. As shown in Fig. 11(a), the values of the $D E T$ at $\theta_{\mathrm{m}}=\pi / 6$ vary between 0.034 and 0.102 , while the values of $D E T$ for other increasing $\theta_{\mathrm{m}}$ are in the range of $0.033-0.053,0.029-0.049$ and $0.033-0.039$, respectively.

As shown in Fig. 11(b) and Fig. 12(b), the variables at $\theta_{\mathrm{m}}=\pi / 4$ and $\pi / 3$ firstly increase and have the maximum at $z / H=0.75$, then significantly decrease for $R e=32940$. The recurrence characteristics at $\theta_{\mathrm{m}}=\pi / 6$ gradually increase with the increase in the $z / H$. This is because the pair of axial flow patterns was gradually induced by the adjacent horizontal jet flow ${ }^{23,24}$. Nevertheless, the values at $\theta_{\mathrm{m}}=$ $\pi / 6$ are smaller than those at others $\theta_{\mathrm{m}}$. As shown in Fig. 12(b), the values of $R R$ with an increasing $\theta_{\mathrm{m}}$ are in the range of $0.022-0.03,0.022-0.075$, $0.047-0.084$ and $0.036-0.051$, respectively. This means that the fluid characteristic of PFS at $\theta_{\mathrm{m}}=\pi / 6$ for $R e=32940$ have poor periodicity, predictability and weak recurrence compared with those at others $\theta_{\mathrm{m}}$. It can be concluded from Figs. 11(b)-13(b) that the recurrence characteristics of PFS at $\theta_{\mathrm{m}}=\pi / 3$ is the strongest, and then that at $\theta_{\mathrm{m}}=5 \pi / 12$ ranks the second among four $\theta_{\mathrm{m}}$ for $R e=32940$.

Compared with the subplots of recurrence characteristics of PFS between lower and higher Re, there are some distinguishing differences. The results as described in Figs. 11(a)-13(a) indicate that 

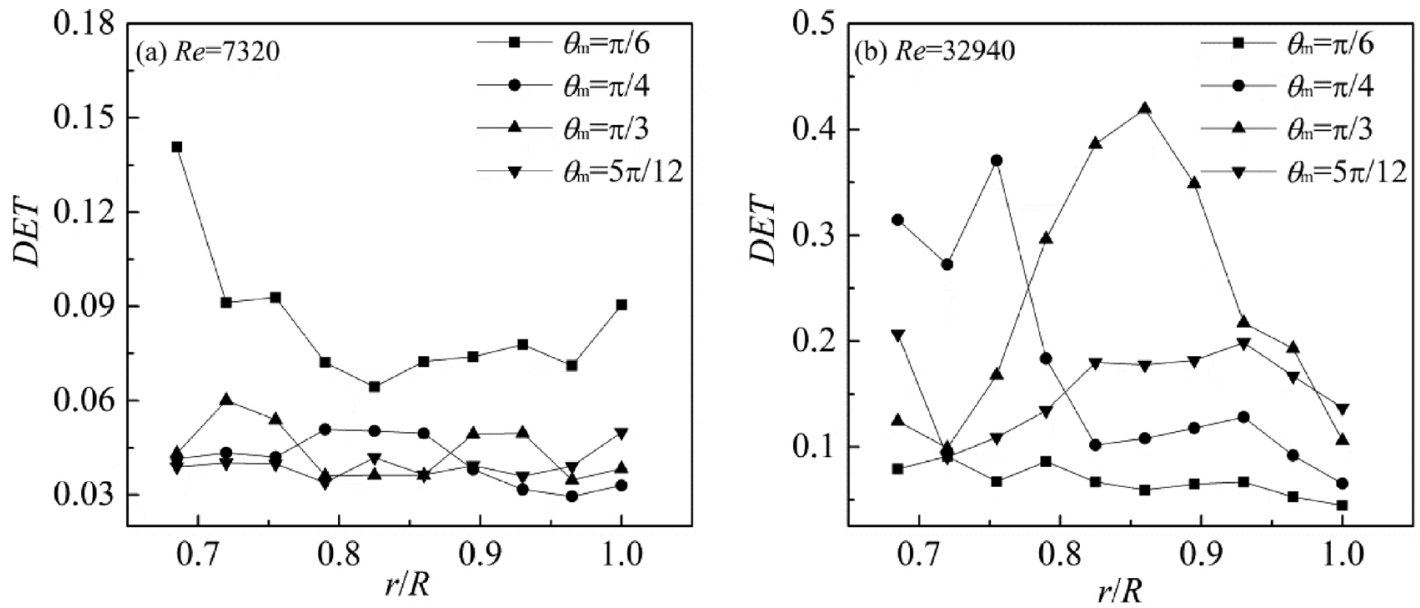

Fig. 8 -Determinism of the PFS vs. r/R
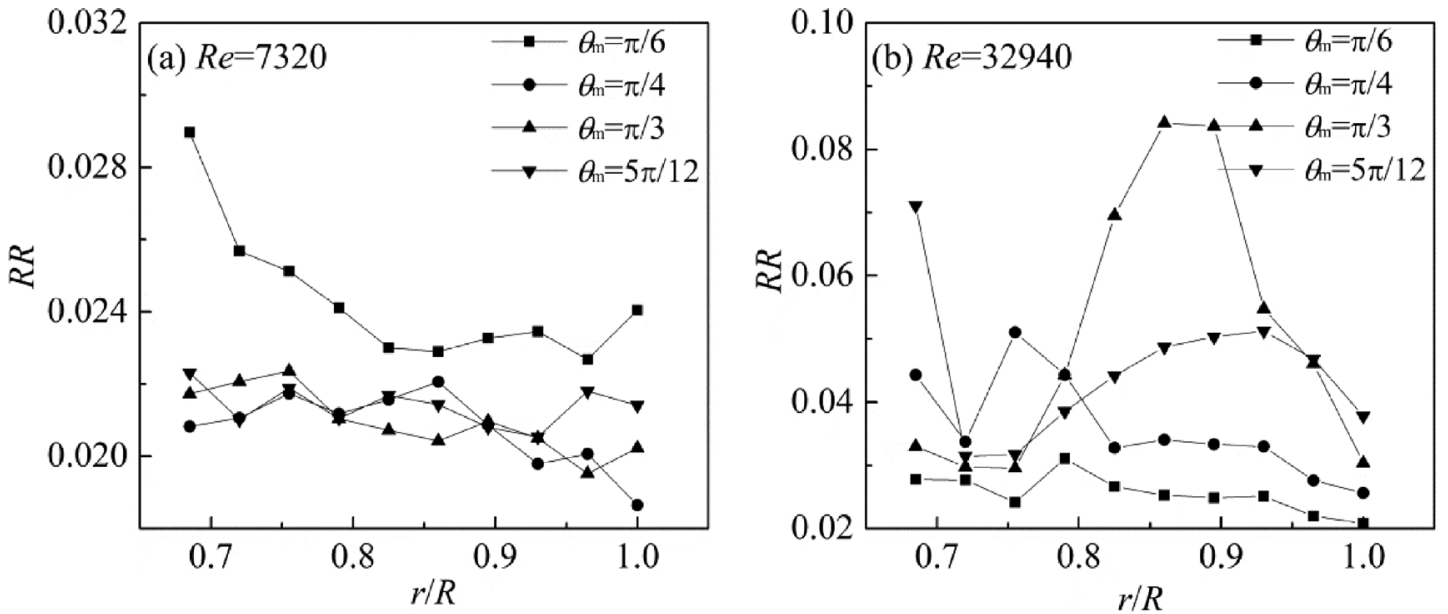

Fig. 9 - Recurrence rate of the PFS vs. $r / R$
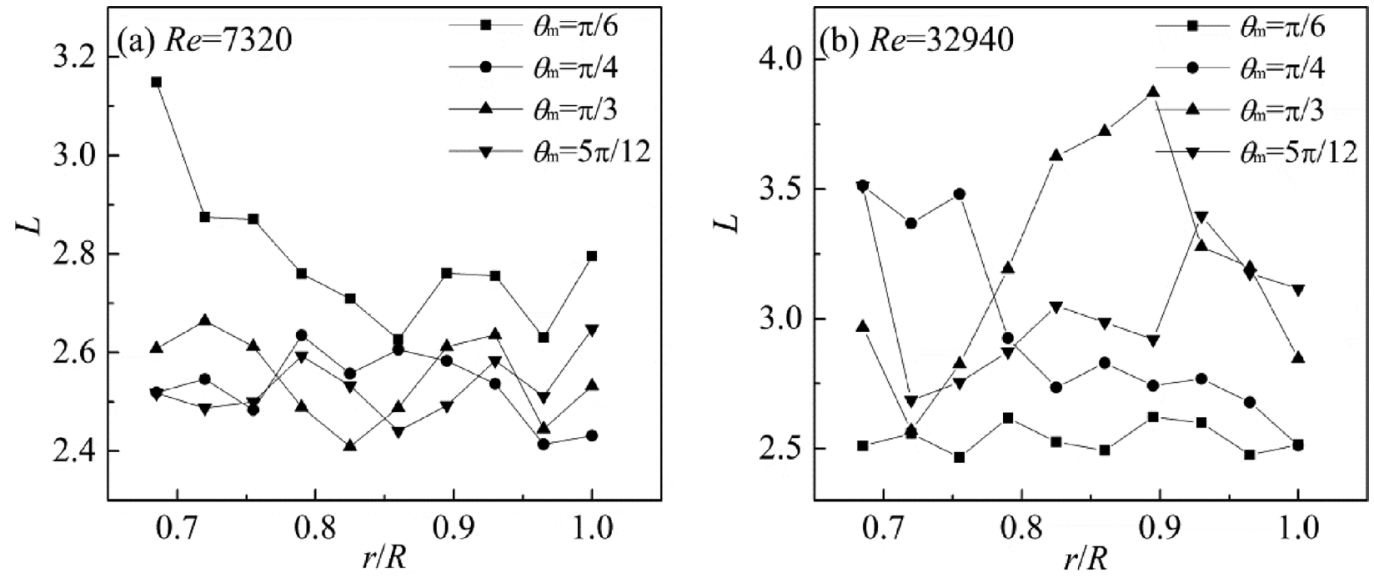

Fig. 10 -Average diagonal line length of the PFS vs. $r / R$

the jet velocity from the nozzle declines quickly along the jet length and there is not enough energy to transfer between different scale vortices at $\theta_{\mathrm{m}}>\pi / 6$ for the lower Re. While the jet flow becomes stronger at $R e=32940$, the effect of the axial flow pattern is gradually generated between the vertical adjacent jet flow at $\theta_{\mathrm{m}}=\pi / 6$. At the same time, the higher turbulent kinetic energy from the horizontal nozzle can be transferred a longer distance. As a result, much larger scale vortices are induced with higher determinism at $\theta_{\mathrm{m}}=\pi / 4$ and $\theta_{\mathrm{m}}=\pi / 3$. 

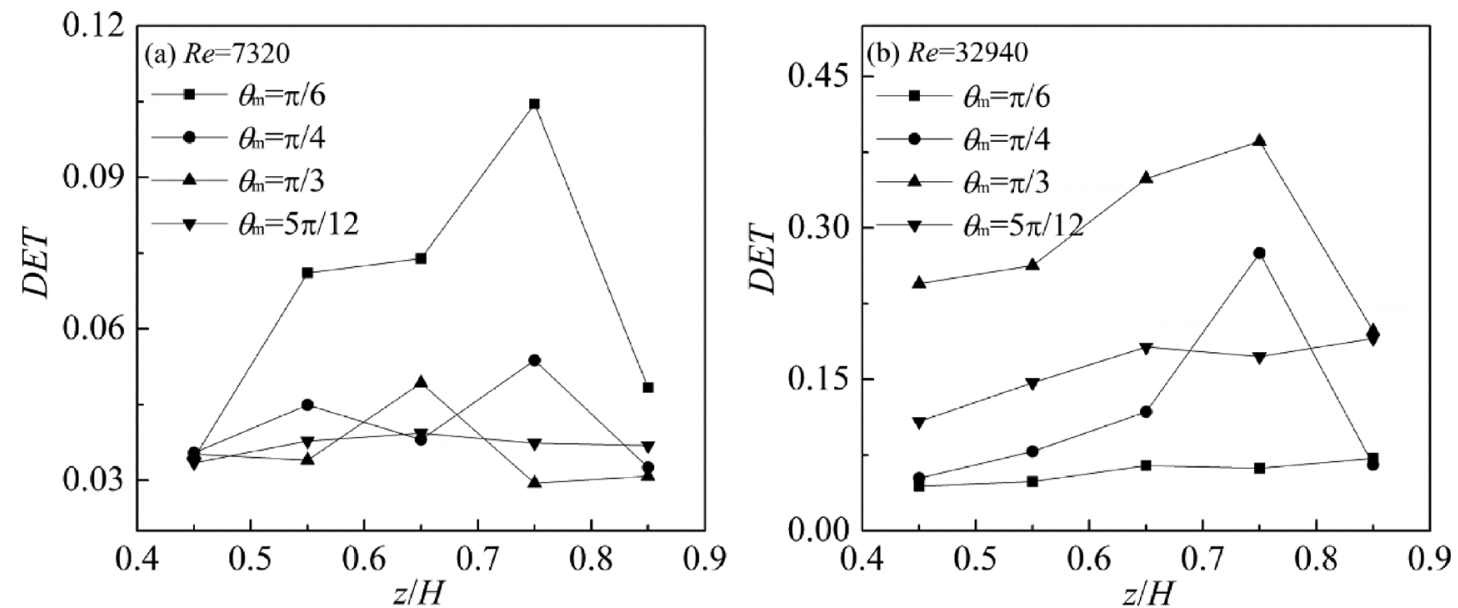

Fig. 11 -Relationship between DET and axial positions at different $\theta_{m}$
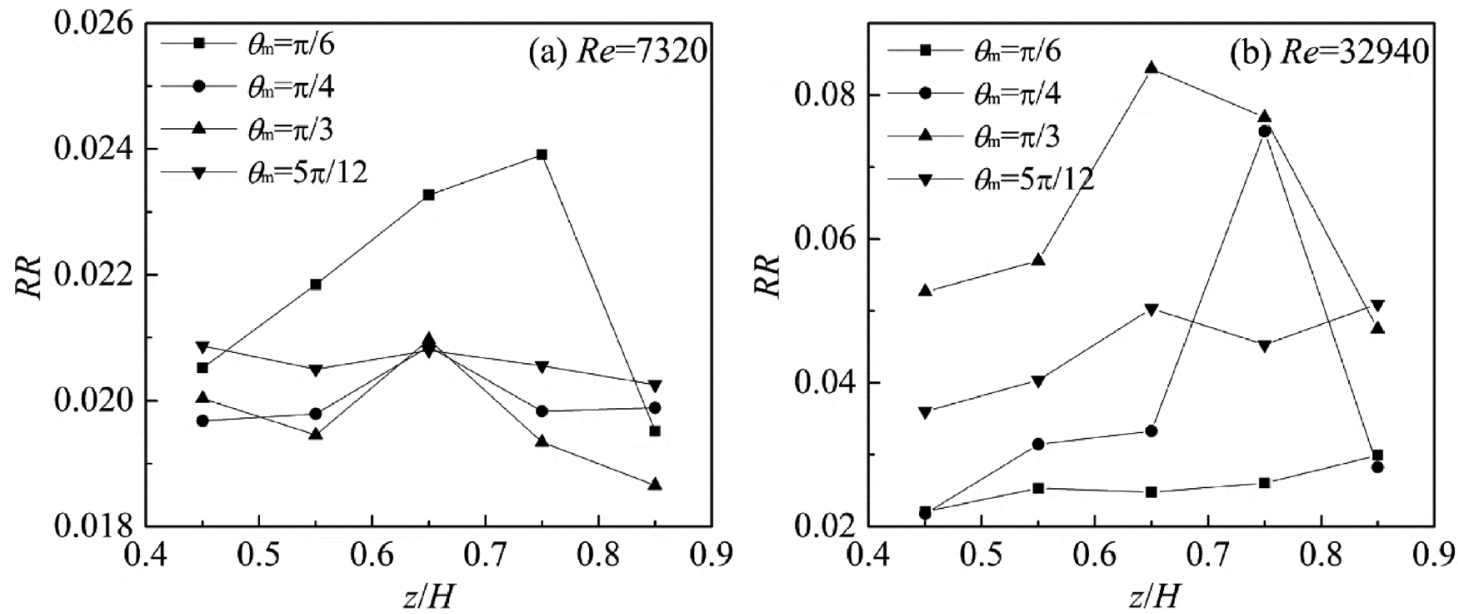

Fig. 12 - Relationship between RR and axial positions at different $\theta_{m}$
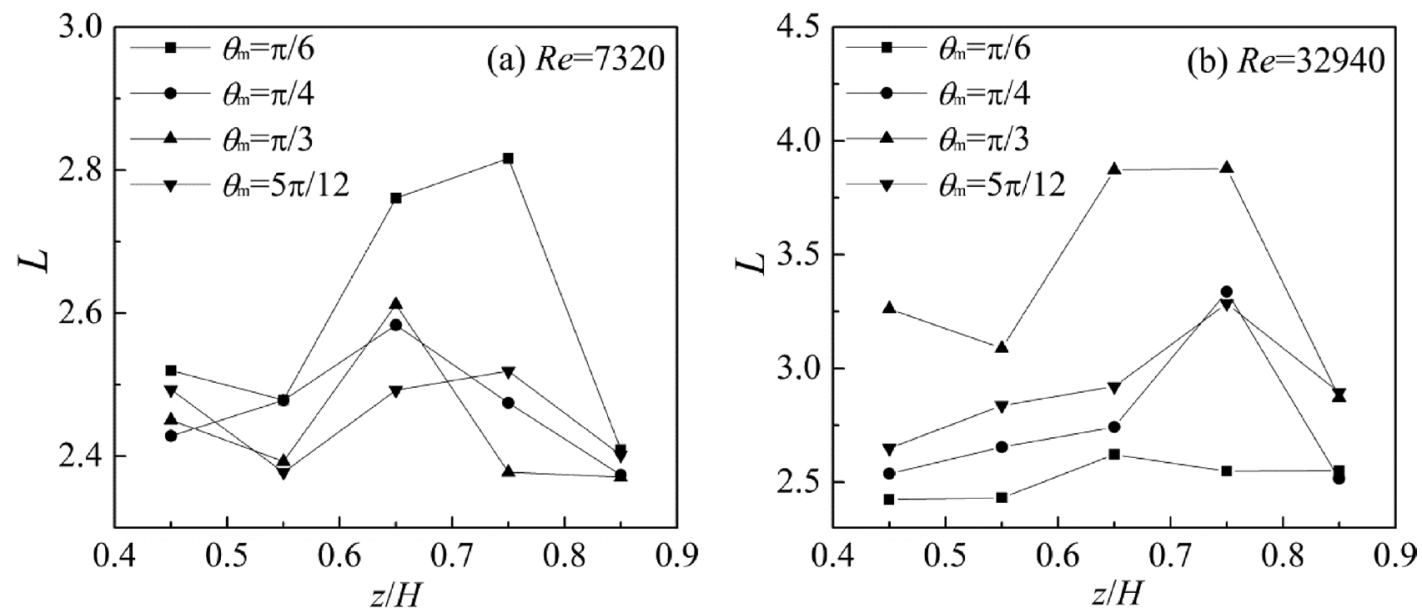

Fig. 13 -Relationship between $L$ and axial positions at different $\theta_{m}$

\section{Conclusion}

In this work, RPs and RQA were employed to reveal the recursion phenomenon of PFS in the novel CJT. The RQA parameters were studied at different Reynolds numbers, circumferential and axial locations. It is revealed that the determinism of RP increases and the predictability of PFS becomes greater with the increasing Re. The RQA variables linearly increase, then significantly increase, and at last abruptly decrease with the increasing $\theta_{\mathrm{m}}$. The recursion characteristics decrease firstly, then sharp- 
ly increase, and decrease at last with the increasing $r / R$ except for $\theta_{\mathrm{m}}=\pi / 6$. With the increasing $z / H$, the RP characteristics of $\theta_{\mathrm{m}}=\pi / 4$ and $\pi / 3$ increase firstly and then decrease. The recurrence characteristics of $\theta_{\mathrm{m}}=\pi / 6$ gradually increase with the increase in the $z / H$, but the values of characteristic variables are smaller than those of other $\theta_{\mathrm{m}}$. The results suggest that RQA can be used as a quantitative tool to evaluate the hydrodynamics of the novel CJT, which provides a referential basis for further optimal design.

\section{ACKNOWLEDGMENTS}

This work was supported by the National Natural Science Foundation of China (Nos. 21476142, 21306115, 21106086), the Program for Liaoning Excellent Talents in University (No. LR2015051), the Natural Science Foundation of the Liaoning Science and Technology Bureau of China (No. 2015020148), the Science Foundation for Doctorate Research of the Liaoning Science and Technology Bureau of China (No.20131090). We also thank the reviewers for their enlightening remarks that helped us both in English, and in depth to improve the quality of the paper.

\section{Nomenclature}

DET - Determinism

$D I V$ - Divergence

$E_{\mathrm{i}} \quad-$ Wavelet decomposition error of PFS for Dau $(i)$

ENTR- Shannon Entropy

$f_{S} \quad$ - Sampling frequency $(\mathrm{Hz})$

$H$ - Liquid height ( $\mathrm{mm}$ )

$l$ - Diagonal line of length

$L \quad$ - Average diagonal line length

$L_{\max }$ - Maximum diagonal line length

$m$ - Embedding dimension

$N \quad$ - Length of time series

$p(l)$ - Probability to find a diagonal line of length $l$

$r \quad$ - Radial location of the measurement point (mm)

$R \quad$ - Radius of the tank ( $\mathrm{mm})$

$R A T I O$ - Ratio between $D E T$ and $R R$

Re - Reynolds number

$R_{\mathrm{i}, \mathrm{j}}^{\mathrm{m}}-$ Recurrence state of the phase space reconstructed

$R R$ - Recurrence rate

std - Standard deviation

$t \quad$ - Time (s)

$x(i)$ - Data points

$z \quad-$ Axial coordination (mm)

\section{Greek letters}

$\alpha \quad-$ Radius to be selected

$\Delta \tau \quad-$ Time interval (s)

$\varepsilon \quad-$ Threshold of distance

$\theta_{\mathrm{m}} \quad$ - Measurement angle

$\Theta(\bullet)$ - Heaviside function

$\tau \quad-$ Time delay (s)

\section{Reference}

1. Sultan, M. A., Krupa, K., Fonte, C. P., Nunes, M. I., Dias, M. M., Lopes, J. C. B., Santos, R. J., High-Throughput T-Jets Mixers: An Innovative Scale-Up Concept, Chem. Eng. Technol. 36 (2013) 323. doi: http://dx.doi.org/10.1002/ceat.201200141

2. Valente, I., Celasco, E., Marchisio, D. L., Barresi, A. A., Nanoprecipitation in Confined Impinging Jets Mixers: Production, Characterization and Scale-Up of Pegylated Nanospheres and Nanocapsules for Pharmaceutical Use, Chem. Eng. Sci. 77 (2012) 217. doi: http://dx.doi.org/10.1016/j.ces.2012.02.050

3. Sundararaj, S., Selladurai, V., The Effects of Arbitrary Injection Angle and Flow Conditions on Venturi-Jet Mixer, Therm. Sci. 16 (2012) 207. doi: http://dx.doi.org/10.2298/tsci101023059S

4. Icardi, M., Gavi, E., Marchisio, D. L., Barresi, A. A., Olsen, M. G., Fox, R. O., Lakehal, D., Investigation of the Flow Field in A Three-Dimensional Confined Impinging Jets Reactor by Means of Micro PIV and DNS, Chem. Eng. J. 166 (2011) 294. doi: http://dx.doi.org/10.1016/j.cej.2010.09.046

5. Lince, F., Marchisio, D. L., Barresi, A. A., A Comparative Study for Nanoparticle Production with Passive Mixers via Solvent-Displacement: Use of CFD Models for Optimization and Design, Chem. Eng. Proce. 50 (2011) 356. doi: http://dx.doi.org/10.1016/j.cep.2011.02.015

6. Voke, P. R., Gao, S., Numerical Study of Heat Transfer from an Impinging Jet, Int. J. Heat Mass Transfer 41 (1998) 671. doi: http://dx.doi.org/10.1016/S0017-9310(97)00243-3

7. Kandakure, M. T., Patkar, V. C., Patwardhan, A. W., Patwardhan, J. A., Mixing with Jets in Cross-Flow, Ind. Eng. Chem. Res. 48 (2009) 6820. doi: http://dx.doi.org/10.1021/ie801863a

8. Fossett, H., Prosser, L. E., The Application of Free Jets to the Mixing of Fluids in Bulk, Proc. Inst. Mech. Eng. 160 (1949) 224.

9. Lehrer, I. H., A New Model for Free Turbulent Jets of Miscible Fluids of Different Densities and a Jet Mixing Time Criterion, Trans. Inst. Chem. Eng. 59 (1981) 247.

10. Simon, M., Fonade, C., Experimental Study of Mixing Performances Using Steady and Unsteady Jets, Can. J. Chem. Eng. 71 (1993) 507. doi: http://dx.doi.org/10.1002/cjce.5450710402

11. Riffat, S. B., Gan, G., Smith, S., Computational Fluid Dynamics Applied to Ejector Heat Pumps, Appl. Ther. Eng. 16 (1996) 291. doi: http://dx.doi.org/10.1016/1359-4311(95)00033-X

12. Ranade, V. V., Towards Better Mixing Protocols by Designing Spatially Periodic Flows-The Case of a Jet Mixer, Chem. Eng. Sci. 51 (1996) 2637. doi: http://dx.doi.org/10.1016/0009-2509(96)00129-7 
13. Jayanti, S., Hydrodynamics of Jet Mixing in Vessels, Chem. Eng. Sci. 56 (2001) 193 doi: http://dx.doi.org/10.1016/S0009-2509(99)00588-6

14. Zughbi, H. D., Rakib, M. A., Investigation of Mixing in A Fluid Jet Agitated Tank, Chem. Eng. Commun. 189 (2002) 1038. doi: http://dx.doi.org/10.1080/00986440213878

15. Patwardhan, A.W., Thatte, A. R., Process Design Aspects of Jet Mixers, Can. J. Chem. Eng. 82 (2004) 198. doi: http://dx.doi.org/10.1002/cjce.5450820126

16. Gavi, E., Marchisio, D. L., Barresi, A. A., CFD Modelling and Scale-up of Confined Impinging Jet Reactors, Chem. Eng. Sci. 62 (2007) 2228. doi: http://dx.doi.org/10.1016/j.ces.2006.12.077

17. Parvareh, A., Rahimi, M., Alsairafi, A. A., Experimental and CFD Studies on the Effect of the Jet Position on Mixing Performance, Iran. J. Chem. Eng. 6 (2009) 3.

18. Patwardhan, A. W., Gaikwad, S. G., Mixing in tanks agitated by jets, Chem. Eng. Res. Des. 81 (2003) 211. doi: http://dx.doi.org/10.1205/026387603762878674

19. Raja, T., Kalaichelvi, P., Anantharaman, N., Development of CFD for Optimum Mixing in Jet Mixed Tanks, J. Sci. Ind. Res. 66 (2007) 522.

20. Zughbi, H. D., Numerical Simulation of Mixing in A Jet Agitated Horizontal Cylindrical Tank, Int. J. Comput. Fluid. Dyn. 20 (2006) 127. doi: http://dx.doi.org/10.1080/10618560600703736

21. Zughbi, H. D., Ahmad, I., Mixing in Liquid-Jet-Agitated Tanks: Effects of Jet Asymmetry, Ind. Eng. Chem. Res. 44 (2005) 1052. doi: http://dx.doi.org/10.1021/ie0496683

22. Meng, H. B., Wang, W., Wu, J. H., Yu, Y. F., Wang, F., Experimental Study on Instantaneous Pressure Fluctuation Time Series in the Novel Tank Agitated by Multiple Horizontal Jets, Chem. Eng. Res. Des. 90 (2012) 1750. doi: http://dx.doi.org/10.1016/j.cherd.2012.04.002

23. $Y u, Y . F ., W u, J . H .$, Meng, H. B., Numerical Simulation Process Aspects of the Novel Static Circulating Jet Mixer, Can. J. Chem. Eng. 89 (2011) 460. doi: http://dx.doi.org/10.1002/cjce.20433

24. Yu, Y. F., Wu, J. H., Meng, H. B., Turbulent Characteristics of A Novel Circulating Jet Mixer, Chin. J. Process Eng. 11 (2011) 1

25. Faure, P., Korn, H., A New Method to Estimate the Kolmogorov Entropy from Recurrence Plots: Its Application to Neuronal Signals, Physica. D. 122 (1998) 265 doi: http://dx.doi.org/10.1016/S0167-2789(98)00177-8

26. Kantz, H., Schreiber, T., Nonlinear Time Series Analysis (2 edition), Cambridge University Press, Cambridge, UK, 2004.

27. Amoud, H., Agrawal, M., Doussot, M., Duchene, J., Intrinsic Mode Entropy for Nonlinear Discriminant Analysis, IEEE Signal Processing Letters 14 (2007) 297. doi: http://dx.doi.org/10.1109/1sp.2006.888089

28. Llauró, F. X., Llop, M. F., Characterization and Classification of Fluidization Regimes by Non-Linear Analysis of Pressure Fluctuations, Int. J. Multiphase Flow 2 (2006) 1397.

doi: http://dx.doi.org/10.1016/j.ijmultiphaseflow.2006.06.011

29. Marwan, N., Romano, M. C., Thiel, M., Jürgen, K., Recurrence Plots for the Analysis of Complex Systems, Phys. Rep. 438 (2007) 237. doi: http://dx.doi.org/10.1016/j.physrep.2006.11.001
30. Meng, H. B., Wang, F., Yu, Y. F., Wang, Y. F., Wu, J. H., Multiscale Entropy Analysis of Instantaneous Pressure Fluctuation in the Novel Jet Tank, Chem. Eng. Technol. 36 (2013) 2137. doi: http://dx.doi.org/10.1002/ceat.201200727

31. Yu, Y. F., Xiong, Q., Meng, H. B., Wang, F., Wu, J. H., Characteristics of Chaotic Dynamic Structure Catastrophe of Instantaneous Pressure Signals in A Kenics Static Mixer, Chin. J. Process. Eng. 13 (2013) 368.

32. Perc, M., Nonlinear Time Series Analysis of the Human Electrocardiogram, Eur. J. Phys. 26 (2005) 757. doi: http://dx.doi.org/10.1088/0143-0807/26/5/008

33. Eckmann, J. P., Kamphorst, S. O., Ruelle, D., Recurrence Plots of Dynamical Systems, Europhys. Lett. 4 (1987) 973. doi: http://dx.doi.org/10.1209/0295-5075/4/9/004

34. Marwan, $N$., How to Avoid Potential Pitfalls in Recurrence Plot Based Data Analysis, Int. J. Bifurcat. Chaos 21 (2011) 1003 . doi: http://dx.doi.org/10.1142/S0218127411029008

35. Marwan, N., Donges, J. F., Zou, Y., Donner, R. V., Kurths, $J$., Complex Network Approach For Recurrence Analysis Of Time Series, Phys. Lett. A 373 (2009) 4246. doi: http://dx.doi.org/10.1016/j.physleta.2009.09.042

36. Wang, C. H., Zhong, Z. P., E, J. Q., Flow Regime Recognition in Spouted Bed Based on Recurrence Plot Method, Powder Technol. 219 (2012) 20. doi: http://dx.doi.org/10.1016/j.powtec.2011.11.051

37. Zbilut, J. P., Webber, C. L., Embeddings and Delays as Derived From Quantification of Recurrence Plots, Phys. Lett. A. 171 (1992) 199 doi: http://dx.doi.org/10.1016/0375-9601(92)90426-M

38. Zbilut, J. P., Webber, C. L., Dynamical Assessment of Physiological Systems and States Using Recurrence Plot Strategies, J. Appl. Physiol. 76 (1994) 965.

39. Li, S. C., Liu, F. Y., Dai, E. F., Wu, S. H., Recurrence Quantification Analysis for Geographical Differences of the Coupling Relationship Between NDVI and Climatic Factors, Acta Scientiarum Naturalium Universitais Pekinensis China 44 (2008) 483

40. Zou, Y., Donner, R. V., Donges, J. F., Marwan, N., Kurths, $J$., Identifying Complex Periodic Windows in Continuous-time Dynamical Systems Using Recurrence-based Methods, Chaos 20 (2010) 043130. doi: http://dx.doi.org/10.1063/1.3523304

41. Meng, H. B., Wang, F., Yu, Y. F., Wang, W., Wu, J. H., Delay Time Correlation of Pressure Fluctuation Signals in the Novel Circulating Jet Tank, Chem. Biochem. Eng. Q. 27 (2013) 251.

42. Christian, G., Esterina, M., Karine, T., Estimating Copula Densities through Wavelets, Insur. Math. Econ. 44 (2009) 170. doi: http://dx.doi.org/10.1016/j.insmatheco.2008.07.006

43. Nelson, J. D. B., Kingsbury, N. G., Dual-tree Wavelets for Estimation of Locally Varying and Anisotropic Fractal Dimension, ICIP, 2010. doi: http://dx.doi.org/ 10.1109/ICIP.2010.5653915

44. Saavedra-Gastélum, V., Rivera, A. L., Fernández-Harmony T., Castaño, E., Castaño, V. M., Signals from Living Biomaterials: Analysis of Human Brain Signals through Wavelets, Mater. Res. Innovations 14 (2010) 247. doi: http://dx.doi.org/10.1179/143307510X12719005364783 
45. Genovese, L., Videau, B., Ospici, M., Deutsch, T., Goedecker, S., Méhaut, J. F., Daubechies Wavelets for High Performance Electronic Structure Calculations: the BigDFT Project, CR. Mécanique 339 (2011) 149.

doi: http://dx.doi.org/10.1016/j.crme.2010.12.003

46. Marwan, N., Schinkel, S., Kurths, J., Recurrence Plots 25 Years Later - Gaining Confidence In Dynamical Transitions, EPL 101 (2013) 20007

doi: http://dx.doi.org/10.1209/0295-5075/101/20007
47. Schinkel, S., Dimigen, O., Marwan, N., Selection of Recurrence Threshold for Signal Detection, Eur. Phys. J. Special Topics 164 (2008) 45. doi: http://dx.doi.org/ 10.1140/epjst/e2008-00833-5

48. Babaei, B., Zarghami, R., Sotudeh-Gharebagh, R., Monitoring of Fluidized Beds Hydrodynamics Using Recurrence Quantification Analysis, AIChE J. 59 (2013) 399.

doi: http://dx.doi.org/ 10.1002/aic.13850 